\title{
The Second Lunar Anomaly in Ancient Indian Astronomy
}

\author{
Dennis W. Duke, Florida State University
}

All early Indian lunar models, from about A.D. 450 - 650, used only one anomaly - the variation in speed of the Moon as it circles the zodiac. ${ }^{1}$ Unlike the Hipparchan model, which treated the same anomaly in terms of a single epicycle or its equivalent eccentric deferent, the Indian lunar (and solar) models without exception used the concentric equant, in which the Earth is at the center of a deferent with radius $R=1$ and the center of uniform motion is some distance $r$ from that center (see Figure 1). In such a model the equation of center $q$ is given by

$$
\sin q=-r \sin \alpha
$$

where the mean anomaly $\alpha=L-A, L$ is the Moon's mean longitude, and $A$ the longitude of its apsidal line. ${ }^{2}$ For the concentric equant the apogee - the direction of slowest motion - is in the direction of the line from the earth to the equant point. The quality of the predictions of the concentric deferent and the Hipparchan model are essentially equivalent.

The Laghumanasam, a short text by Munjala probably written around A.D. 930, gives much of the standard Indian planetary model information and appears to be derived from Aryabhata's various texts, written ca. A.D. 500, Brahmagupta's Brahmasphutasiddhanta, ca. A.D. 628, and the Suryasiddhanta, ca. sixth century A. D. ${ }^{3}$ The Laghumanasam is a type of text known as a karana, which is a short work giving simplified and approximate rules for computing astronomical items. Among the rules that Munjala gives is a correction to the equation of center for the Moon in the form

$$
-r^{\prime} \cos \psi \sin \eta
$$

where $\psi=L^{\prime}-A$ is the elongation of the mean Sun from the lunar apsidal line, and $\eta=L-L^{\prime}$ is the elongation of the mean Moon from the mean Sun. Yallaya, in a commentary to the Laghumanasam written in A.D. 1428, claims that this correction was given earlier by Vatesvara (ca. A.D. 904), but that earlier text has not been found and the claim cannot be checked. What is most interesting is that this correction is closely related to the second inequality of lunar motion known to Ptolemy, and known today as evection. The first two terms in modern lunar theory are conventionally written as

$$
-2 e \sin \alpha-\varepsilon \sin (2 \eta-\alpha)
$$

with $2 e=6.29, \varepsilon=1.27$. The first term is the elliptic inequality and the second is the evection. Noting that $\alpha=\eta+\psi$ and $2 \eta-\alpha=\alpha-2 \psi$, we have the identity

$$
-\sin (2 \eta-\alpha)=\sin \alpha-2 \cos \psi \sin \eta
$$


and so the two modern terms can be rewritten as

$$
\begin{aligned}
-2 e \sin \alpha-\varepsilon \sin (2 \eta-\alpha) & =-2 e \sin \alpha+\varepsilon \sin \alpha-2 \varepsilon \cos \psi \sin \eta \\
& =-(2 e-\varepsilon) \sin \alpha-2 \varepsilon \cos \psi \sin \eta \\
& =-r \sin \alpha-r^{\prime} \cos \psi \sin \eta \\
& =-5.02 \sin \alpha-2.55 \cos \psi \sin \eta
\end{aligned}
$$

exactly the same form found in the Laghumanasam. Munjala's numerical values imply a maximum value of about $2 ; 29^{\circ}$ for the second term in the final line, compared say to Ptolemy's $2 ; 40^{\circ}$ and the modern $2 ; 33^{\circ}$. The range of error for Munjala's model is noticeably better than the error range for the full Almagest model (see Figures 2 and 3). ${ }^{4}$

Munjala's expression is interesting on at least two other counts. First, unlike the modern theoretical expression, in which the evection term $-\sin (2 \eta-\alpha)$ does not vanish at syzygy, Munjala's expression neatly isolates from the evection the part that contributes only away from syzygy. Second, the empirical lunar anomaly that remains after accounting for the principal term $-r \sin \alpha$ can be written as

$$
\lambda-L-(-r \sin \alpha)
$$

where $\lambda$ is the lunar longitude, and this can be determined by a series of measurements of $\lambda$ (or, in our case, calculations using modern theory), and is the empirical target that needs explanation by changing the simple lunar model to something more complicated. Of the three possible pair-wise combinations of the variables $\alpha, \eta$, and $\psi$, Munjala's term is expressed in terms of the pair $(\eta, \psi)$ that happens to give the simplest empirical representation of the lunar anomaly after accounting for the first anomaly (see Figures 46 ). Whether the person responsible for the theory was aware of any of this, and the extent to which it played any role in the development of the theory, is, of course, impossible to determine based on the scant information we have.

No ancient Indian astronomy text gives a geometrical figure of any model or discusses the derivation of any model. So while it could be the case that Munjala's theoretical expression is derived from some Greek geometrical model of which we are already aware, such as the crank model given in the Almagest, it seems just as likely, if not more so, that Munjala's expression is in fact an exact consequence of a simple underlying geometrical model, which may or may not have even been known to Munjala (or Vatesvara), recalling that under the Pingree - van der Waerden Hypothesis, the Indian texts summarize centuries old traditions inherited from the Greeks. ${ }^{5}$ Without further information we cannot say whether the model was developed in India or in some earlier, and unknown to us, Greco-Roman tradition.

If the Laghumanasam expression was derived as an exact consequence of some underlying geometrical model, then the model required is straightforward to infer. We start with a concentric equant of eccentricity $2 e$ and imagine that the equant point 
oscillates on a small epicycle of radius $\varepsilon$ centered on the mean equant position (see Figure 7). The angle between the rotating equant point and the mean lunar apsidal line is assumed to be $2 \psi$, so the period of rotation is about seven months. The effect of this model is that the equant eccentricity $\rho$ oscillates between $2 e-\varepsilon$ and $2 e+\varepsilon$, and the true lunar apsidal line oscillates about the mean apsidal direction by an angle $\delta .^{6}$ Thus at any instant the model is a concentric equant with oscillating eccentricity and apsidal line, ${ }^{7}$ so

$$
\sin q=-\rho \sin (\alpha-\delta)
$$

where

$$
\begin{aligned}
& \rho \cos \delta=2 e+\varepsilon \cos 2 \psi \\
& \rho \sin \delta=\quad \varepsilon \sin 2 \psi
\end{aligned}
$$

and $\delta$ is positive for $2 \psi \leq 180^{\circ}$ and negative for $2 \psi \geq 180^{\circ}$. Then

$$
\begin{aligned}
\sin q & =-\rho \sin (\alpha-\delta) \\
& =-2 e \sin \alpha-\varepsilon \sin (\alpha-2 \psi) \\
& =-2 e \sin \alpha-\varepsilon \sin (2 \eta-\alpha) \\
& =-(2 e-\varepsilon) \sin \alpha-\varepsilon \cos \psi \sin \eta \\
& =-r \sin \alpha-r^{\prime} \cos \psi \sin \eta
\end{aligned}
$$

and so we have exact analytic correspondence between the geometrical model and Munjala's expression. ${ }^{8}$

An equivalent model can also be developed by letting the small epicycle rotate through an angle $2 \eta$ measured from the perigee of the epicycle instead of $2 \psi$ measured from the apogee (see Figure 8). In this case $\delta$ is positive for $2 \eta \leq 180^{\circ}$ and negative for $2 \eta \geq 180^{\circ}$, and we get

$$
\begin{aligned}
& \rho \cos \delta=2 e-\varepsilon \cos 2 \eta \\
& \rho \sin \delta=\quad \varepsilon \sin 2 \eta
\end{aligned}
$$

and

$$
\begin{aligned}
\sin q & =-\rho \sin (\alpha+\delta) \\
& =-2 e \sin \alpha+\varepsilon \sin (\alpha-2 \eta) \\
& =-2 e \sin \alpha-\varepsilon \sin (2 \eta-\alpha)
\end{aligned}
$$

matching exactly the result from Figure 7. Therefore the models are equivalent, even though the small epicycle rotates in about seven months in Figure 7 and in about 15 days in Figure 8. Note that the exact equivalence of the two versions depends directly on the fact that for the concentric equant the Earth is always the same distance from the Moon. It is possible, of course, that some more complicated model could give the same expression, but it seems very unlikely that any simpler model could do so. 
The equivalence of the two models, in spite of the different rotation speeds, can also be understood from a simple geometrical analysis. First we need a preliminary result and a simple corollary (see Figure 9):

Theorem. Let EFG be an isosceles triangle, with $\mathrm{EF}=\mathrm{EG}$. From the apex $\mathrm{E}$ drop a line to an arbitrary point $\mathrm{P}$ on the base $\mathrm{FG}$, and extend the line above $\mathrm{E}$ to point $\mathrm{A}$. Let angle $\mathrm{AEF}=\beta$, angle $\mathrm{PEG}=\gamma$, and angle $\mathrm{EPF}=\alpha$. Then $2 \alpha=\beta+\gamma$.

Proof: since the triangle is isosceles, angle EFP = angle EGP. Then application of the fact that the sum of the internal angles of a triangle is $180^{\circ}$ to triangles EFP and EGP establishes the result.

Corollary. Let angle $\beta$ be measured counterclockwise from EA, and let angle $\gamma$ be measured counterclockwise from EP. Let angles $\beta$ and $\gamma$ change uniformly with time so that $\beta=\beta_{0}+\omega_{\beta} t$ and $\gamma=\gamma_{0}+\omega_{\gamma} t$. Then angle EPF $=\alpha$, measured counterclockwise from PE, also changes uniformly with time, and with speed $2 \omega_{\alpha}=\omega_{\beta}+\omega_{\gamma}$.

Now consider a large deferent with center at $\mathrm{O}$ and radius $\mathrm{OM}=R$ (see Figure 10). At a distance $2 e$ from $\mathrm{O}$ place a smaller circle with center $\mathrm{E}$ and radius $\mathrm{EF}=\mathrm{EG}=\varepsilon$. Let angle $\mathrm{AEF}=2 \psi$ and angle $\mathrm{PEG}=2 \eta$, and let $\psi$ and $\eta$ increase uniformly with time at speeds $\omega_{\psi}$ and $\omega_{\eta}$. Then triangle EFG is isosceles and we may apply the Theorem and Corollary. It follows that $\alpha=\eta+\psi$ and the chord FG subtended by angle FEG always makes an angle $\alpha$ with the apsidal line AP, and that $\alpha$ increases uniformly with time with speed $\omega_{\alpha}=\omega_{\psi}$ $+\omega_{\eta}$. Also, if we call OE the mean equant, in both length and direction, and OF and OG two of the true equants, also in both length and direction, then the equation of center $q$ is the same for triangles MOF and MOG. Therefore the geometrical analysis establishes exactly the same equivalence established above using trigonometry. And since the construction is so simple, it seems extremely likely that the equivalence could have been known in antiquity, even at a date far earlier than Munjala's A.D. 932.

The general model has (at least) two interesting special cases. First, if we let $\beta=\gamma=\alpha$ and $\omega_{\beta}=\omega_{\gamma}=\omega_{\alpha}$ then the two radii EF and EG will always point in opposite directions and FEG will be a straight line which always makes an angle $\alpha$ with the apsidal line AP. Thus this special case is exactly the concentric equant used for the first anomaly alone, and we see that one way of understanding the full lunar model is as a generalization of the concentric equant to allow $\beta \neq \gamma$ while keeping $\beta+\gamma=2 \alpha$. Whether this was part of the path to discovery of the second lunar anomaly is, of course, impossible to say without further information.

A second special case has $\beta=0$ and $\omega_{\beta}=0$, and $\gamma=2 \alpha$ and $\omega_{\gamma}=2 \omega_{\alpha}$. Then the radius EF points toward A and stays fixed, and the radius EG rotates counterclockwise with speed $2 \omega_{\alpha}$. This model is then very closely related to the planetary models of Ibn ashShatir (see the discussion below). 
In the general case, with $\beta \neq \gamma$, the point where chord FG crosses the apsidal line AP oscillates up and down, and so we effectively have a pulsating eccentricity, and the pulsation is twice as fast as the motion in anomaly, just as we see in many Indian texts. Thus it could be that when the Indian texts speak of the varying lengths of the eccentricities, they are not saying that we should vary them in the calculation, but rather simply reminding us that their effective lengths are changing. In reality, the Indian texts are so terse, and so generally devoid of explanation of any kind, that this could easily be the case.

Both versions of the model can be changed from concentric deferent to eccentric deferent, and then the equations of center would be given by

$$
\tan q_{\psi}=\frac{-\rho \sin (\alpha-\delta)}{R+\rho \cos (\alpha-\delta)} \quad \text { or } \quad \tan q_{\eta}=\frac{-\rho \sin (\alpha+\delta)}{R+\rho \cos (\alpha+\delta)}
$$

In eccentric deferent models the Earth-Moon distance is the denominator in these expressions and so is, of course, not a constant. Hence the equations of center $q_{\psi}$ and $q_{\eta}$ are not strictly equal, but the differences are small since, as shown above, the numerators are the same, and the differences in the denominators are proportional to the small quantity $r r^{\prime} / R^{2}$. The equivalent double-epicycle version of the eccentric deferent is, of course, the basis of Ibn ash-Shatir's lunar model, ${ }^{9}$ so it is a variant of the Laghumanasam model with (a) exchange of the concentric equant with an eccentre or equivalent large epicycle, (b) counting the angles as in Figure 8, and (c) putting the small epicycle in various places in the figure. One such variant - putting the small epicycle on the tip of the large epicycle - was adopted by Copernicus. ${ }^{10}$ The earliest known Arabic lunar (or planetary) models date to about A.D. 1240, however, so we are not in position to say whether the Laghumanasam model was somehow transmitted to the Arabic astronomers or whether they developed it independently.

If we put the center $\mathrm{E}$ of the small epicycle at some point north of the center of the deferent, and the Earth at some point south of the center, then the various Arabic planetary models are very closely related to the second special case mentioned above. It is well known, of course, that such models are very close approximations to the Ptolemaic equant. ${ }^{11}$ Also, since these models have eccentricities and epicycle radii that are oscillating in both length and direction, they are very similar to the various intermediate steps of the algorithms of Indian planetary models, which also are close approximations to the Ptolemaic equant. ${ }^{12}$ Thus there could be some relation between the Indian models and the double epicycle approximation to the equant - that is, it seems possible that the Indian algorithms are approximations to the equant via some combination of double epicycle implementations of both planetary anomalies.

Although Ptolemy does not discuss the Almagest lunar model in terms of an oscillating eccentricity and apsidal line, the final analytic expression for his crank mechanism plus epicycle model with a shifted apsidal line can be written in the form ${ }^{13}$ 


$$
\tan q=\frac{-r \sin (2 \eta-\delta)}{\rho+r \cos (2 \eta-\delta)}
$$

From the discussion above we know that such a model can always be transformed into an equivalent eccentric model, and in this case the apsidal direction, but not the eccentricity, oscillates around a mean value. The relation of the Almagest model to the Laghumanasam model, if any, is thus obscure.

The eccentric version of the Laghumanasam model has an interesting subsequent history. ${ }^{14}$ Essentially the same model, with the small epicycle rotating through the angle $2 \psi$, was used by Kepler at an intermediate stage of his lunar research, and then abandoned. The model was later used by Philip Lansbergen with the small epicycle rotating through the angle $2 \eta$, and so was essentially the lunar model of ash-Shatir (and hence Copernicus) transformed so that the small epicycle was at the center of the eccentric deferent. Jeremiah Horrocks, post-Kepler but pre-Newton, again used the model in the form of Kepler, with the small epicycle rotating through the angle $2 \psi$, the only significant difference being that for Horrocks the deferent was a Keplerian ellipse and the elliptic inequality respected the equal area in equal time law.

So we see that the physical idea of an oscillating lunar eccentricity and apsidal line was certainly known as early as medieval India and perhaps back to Greco-Roman times. It was used and perhaps re-discovered by Arabic astronomers, by Copernicus, and by various post-Copernican astronomers, including Kepler, van Lansbergen, and Horrocks. And finally, it was the crucial clue Newton borrowed from Horrocks to finally formulate his own lunar model. ${ }^{15}$

A set of illustrative and interactive animations of the models described above may be found online at www.scs.fsu.edu/ dduke/models.htm. ${ }^{16}$ Look for the link to lunar models. 


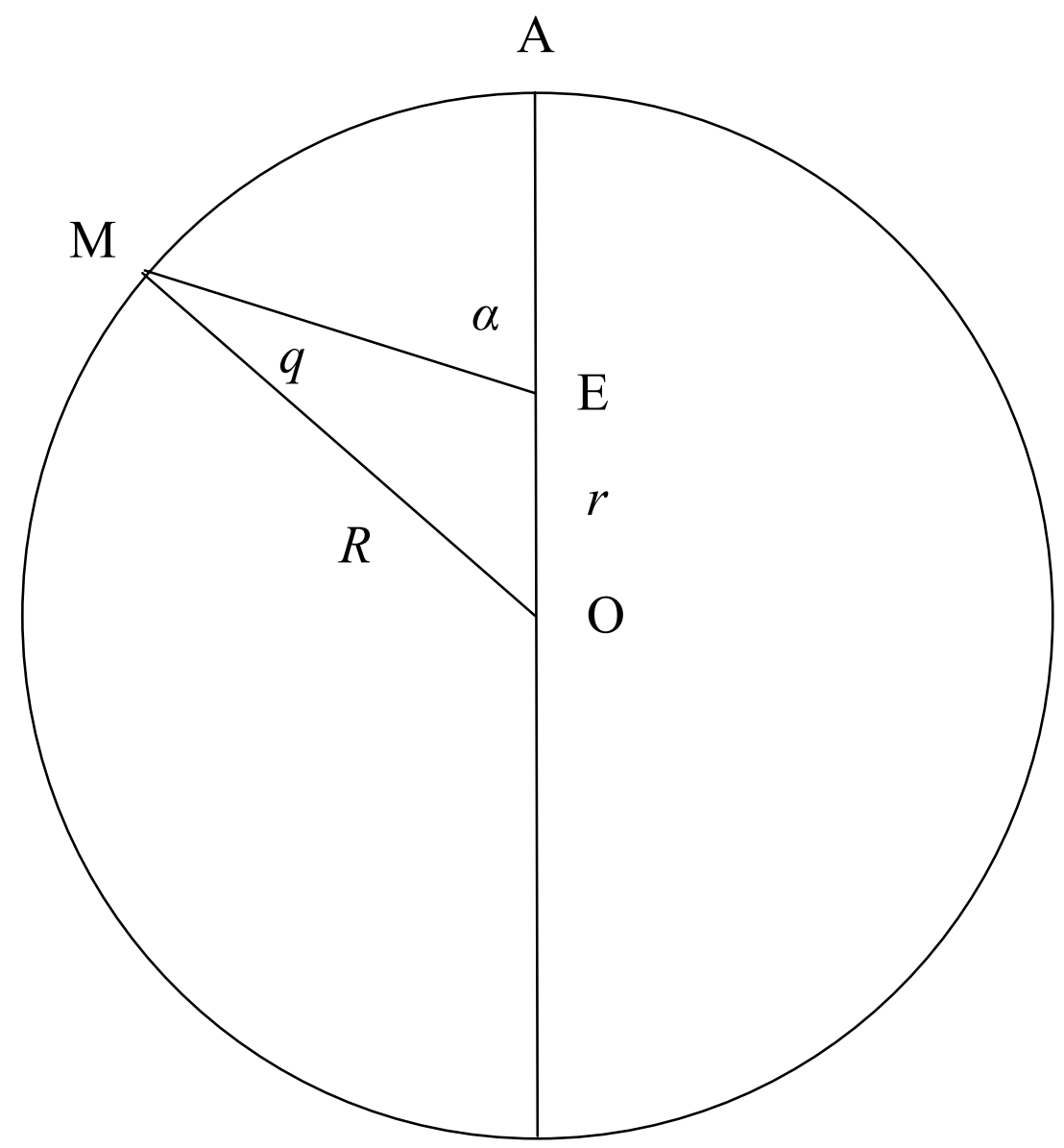

Figure 1. In the concentric equant model the Earth is at the center $\mathrm{O}$ of a deferent of radius $R$ and the moon is at $\mathrm{M}$ on the deferent. The motion of $\mathrm{M}$ is uniform as seen from the equant point $\mathrm{E}$, which is a distance $r$ from $\mathrm{O}$, so angle $\mathrm{AEM}=\alpha$ increases uniformly with time. Angle $\mathrm{OME}=q$ is the equation of center, and is given by $\sin q=-r \sin \alpha$. 


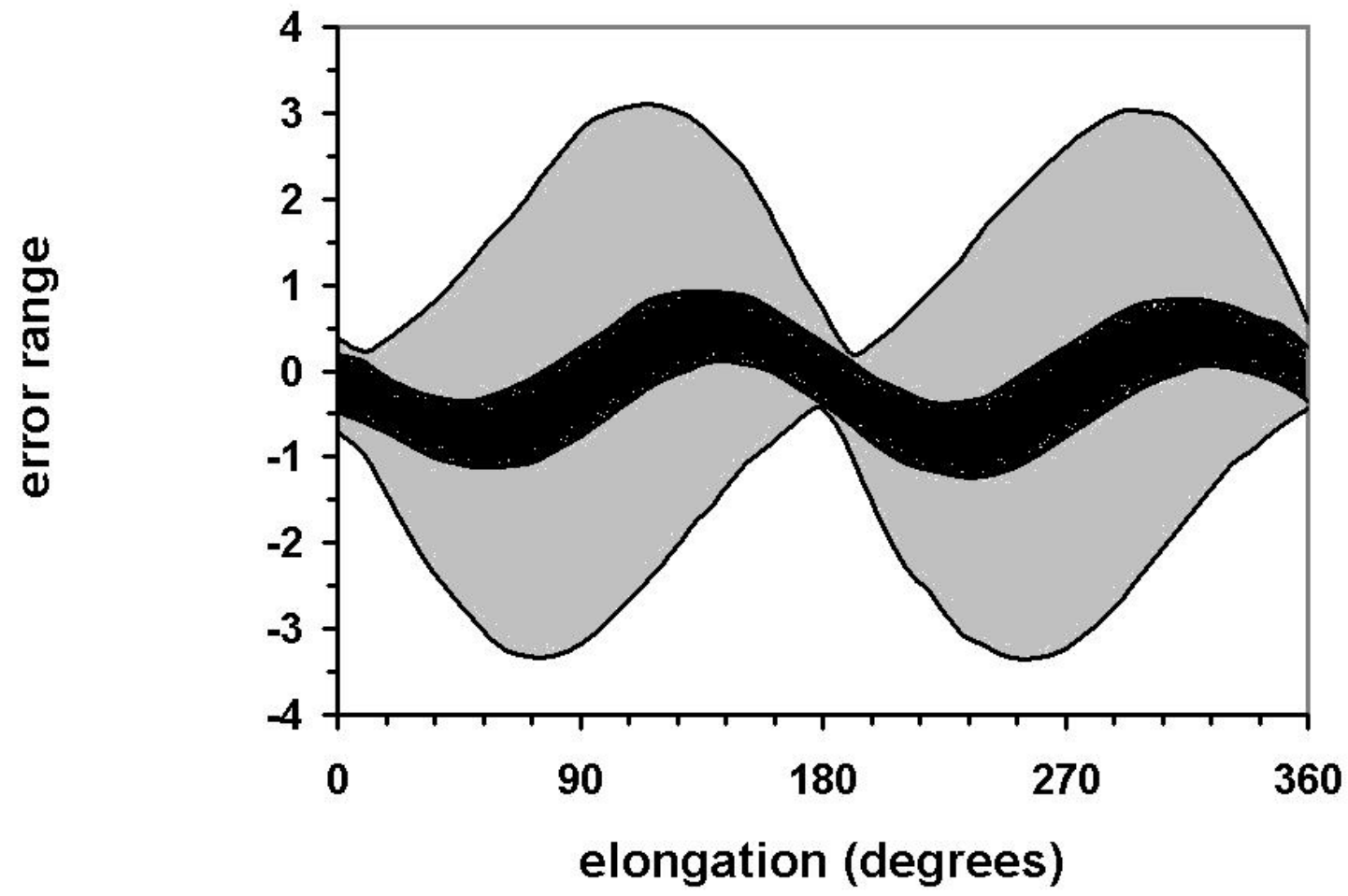

Figure 2. The Indian lunar model error range for the simple model with one anomaly (outer grey area) and the Munjala model (inner black area).

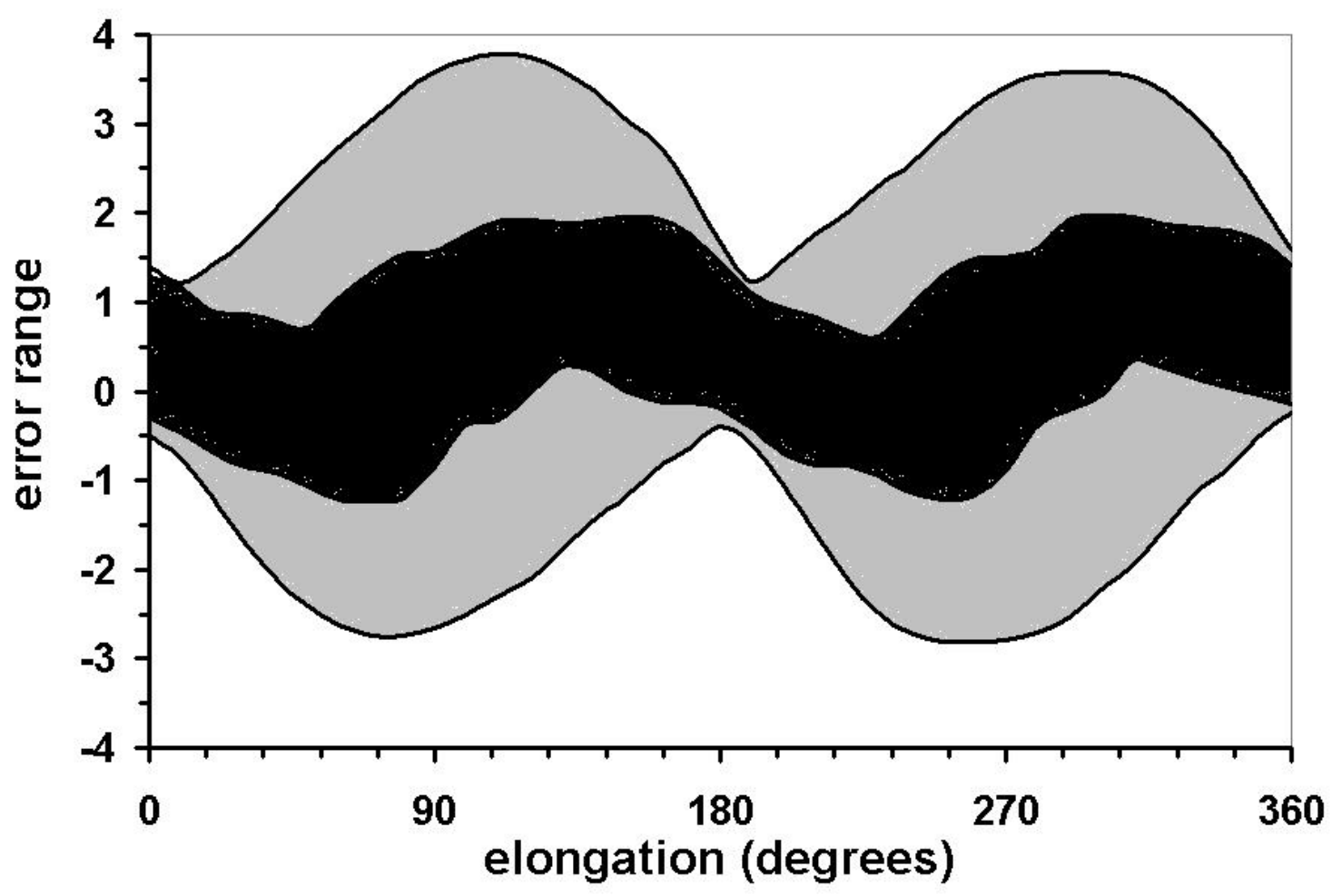

Figure 3. The Almagest lunar model error range for the simple model with one anomaly (outer grey area) and the full model with the crank mechanism and the shifted apogee (inner black area). 


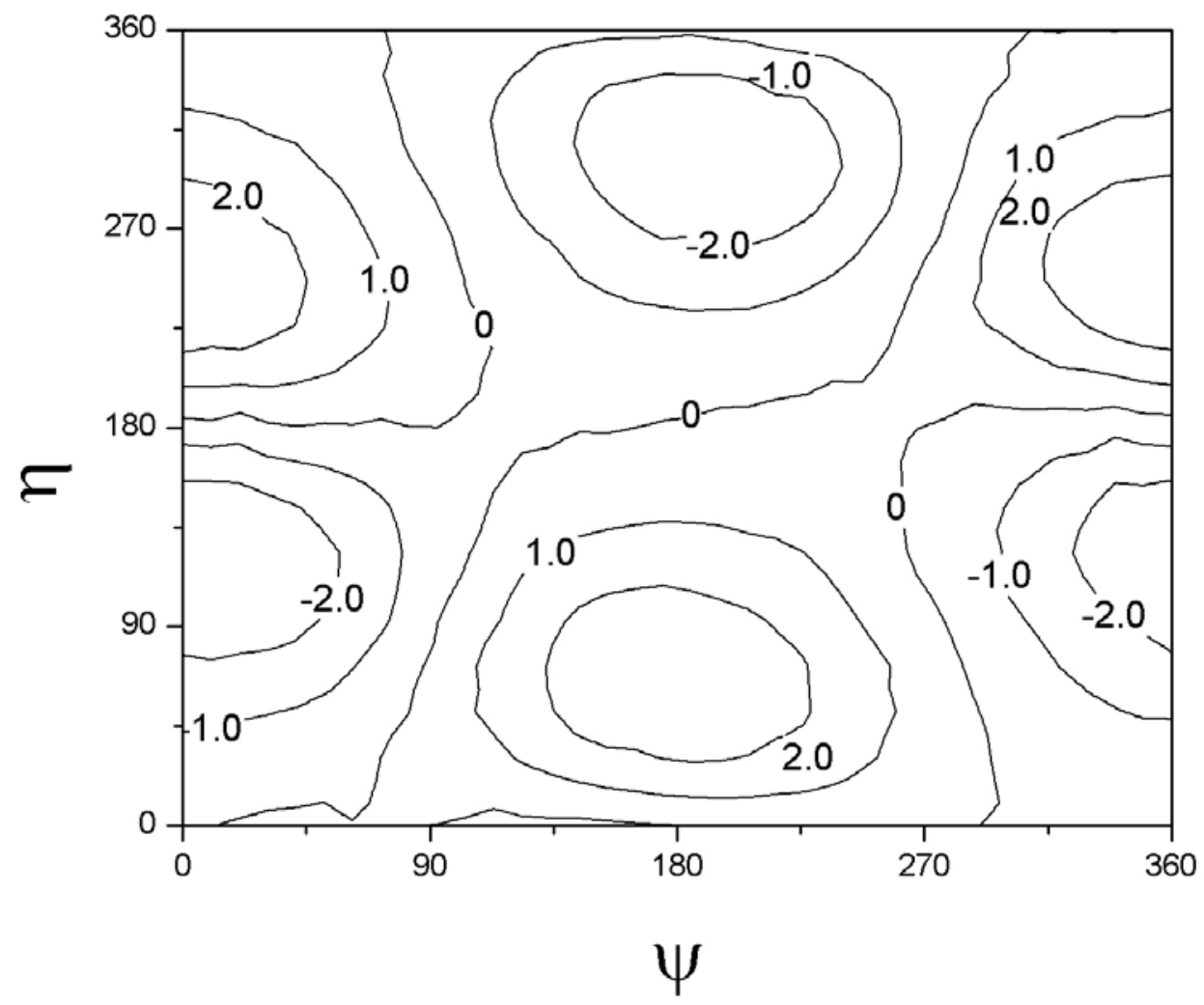

Figure 4.

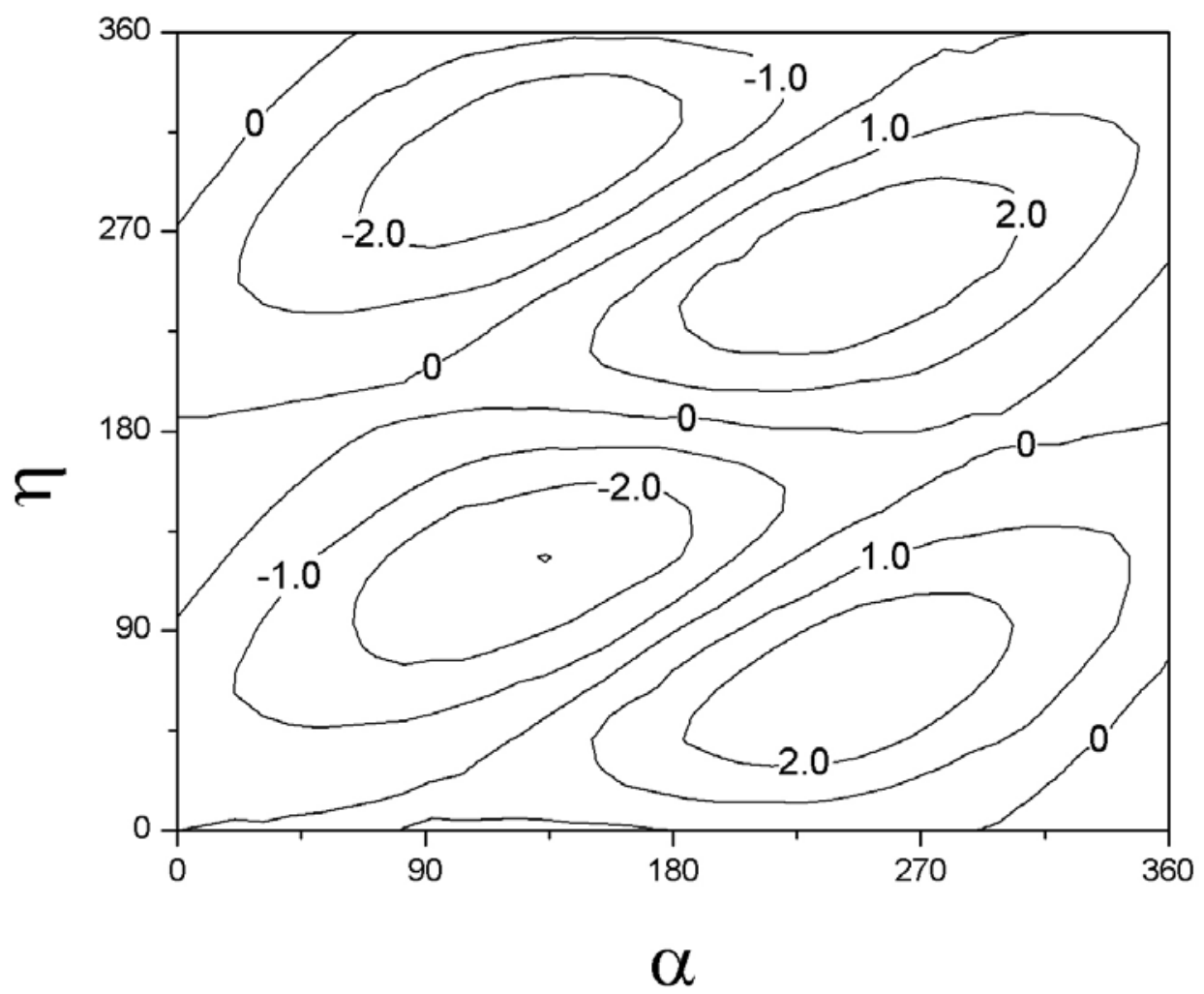

Figure 5. 


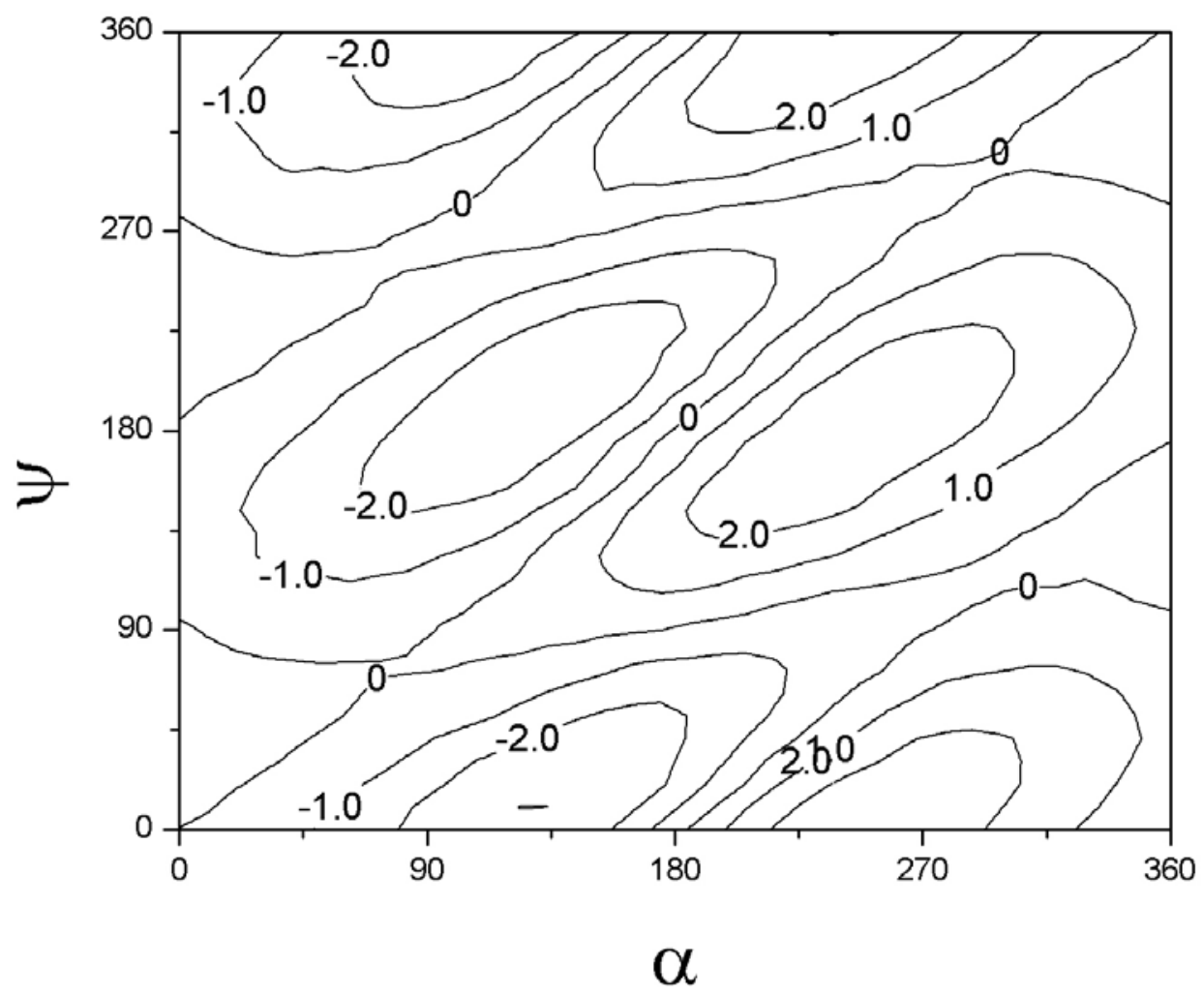

Figure 6. 


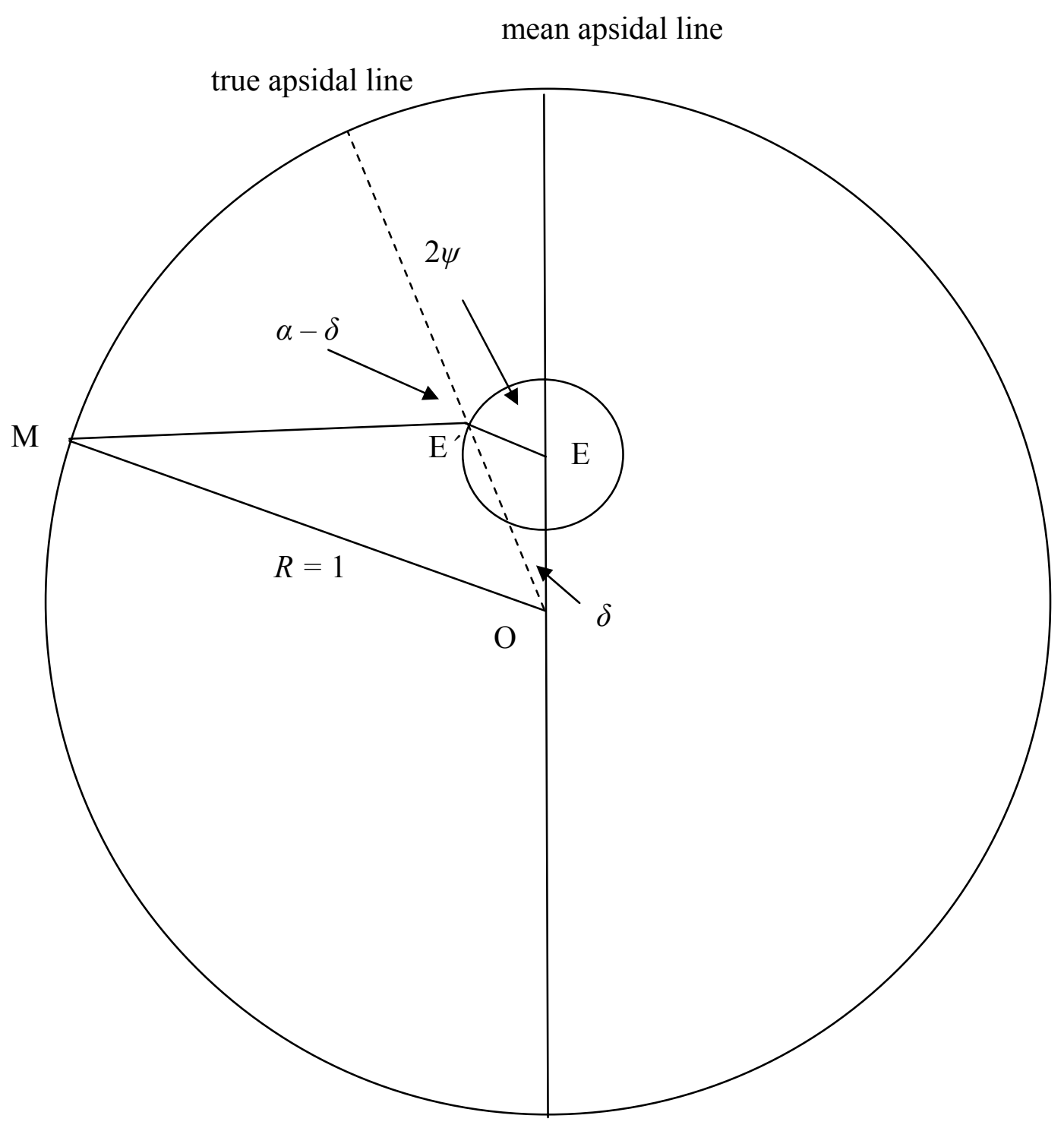

Figure 7. Moon at $\mathrm{M}$, Earth at center $\mathrm{O}$, mean equant at $\mathrm{E}, O E=2 e$, true equant at $\mathrm{E}^{\prime}$, $O E^{\prime}=\rho$. Radius of small epicycle is $\varepsilon$ and rotates counterclockwise. 


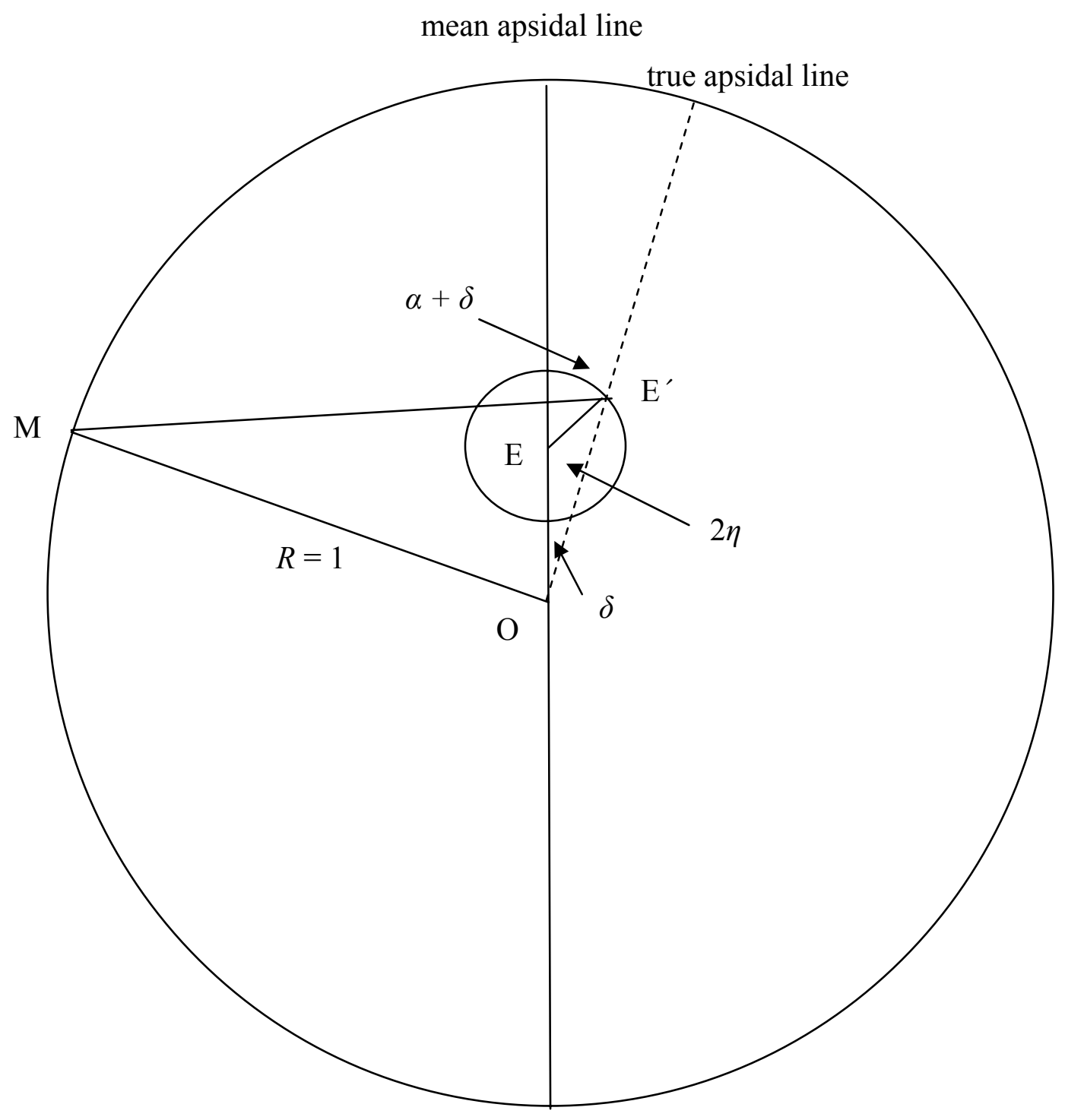

Figure 8. Moon at $\mathrm{M}$, Earth at center $\mathrm{O}$, mean equant at $\mathrm{E}, O E=2 e$, true equant at $\mathrm{E}^{\prime}$, $O E^{\prime}=\rho$. Radius of small epicycle is $\varepsilon$ and rotates counterclockwise. 


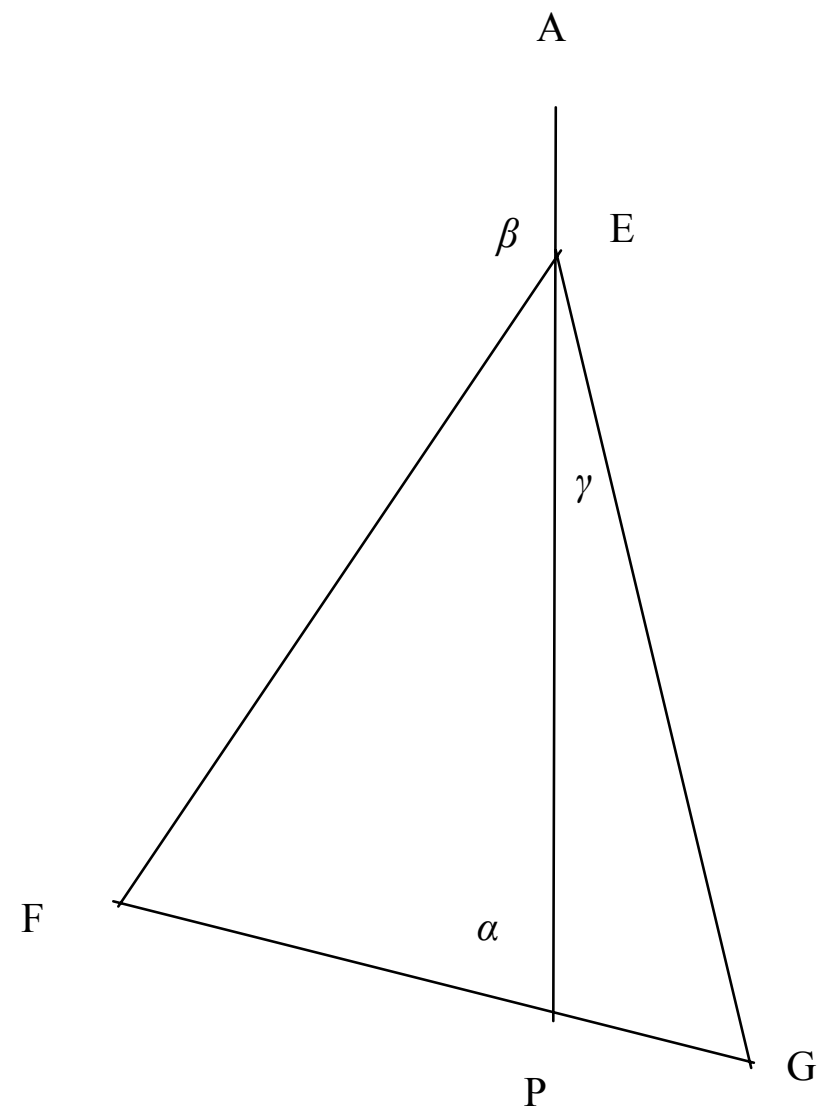

Figure 9. 


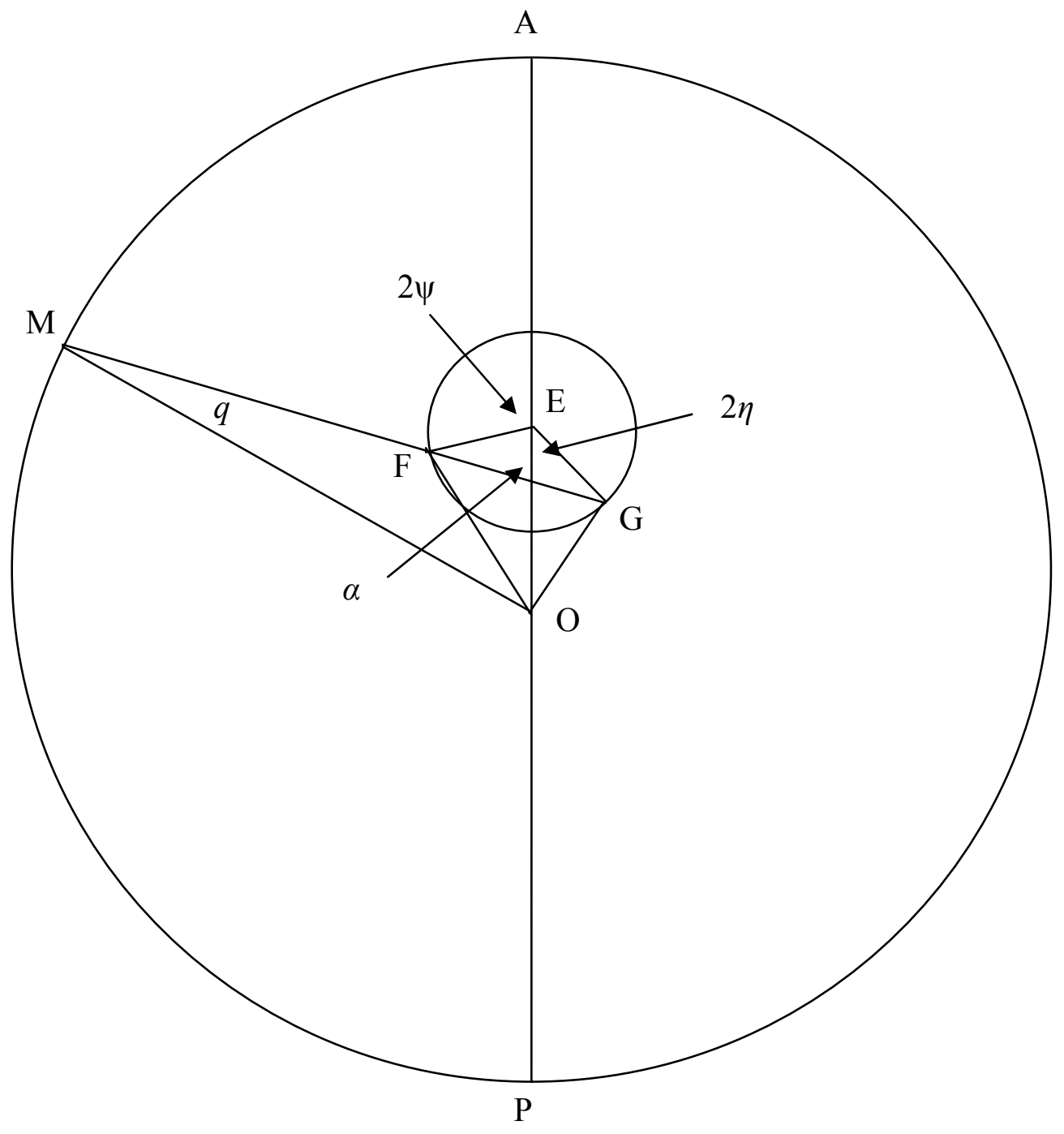

Figure 10. 


\section{REFERENCES}

${ }^{1}$ D. Pingree, "History of Mathematical astronomy in India", Dictionary of Scientific Biography, 15 (1978), 533-633.

${ }^{2}$ D. Pingree, "Concentric with Equant", Archives Internationales d'Histoire des Sciences, 24 (1974) 26-28.

${ }^{3}$ K. S. Shukla, A Critical Study of the Laghumanasa of Manjula, (New Delhi, 1990); D. Pingree, ibid. (ref. 1) 618-619.

${ }^{4}$ These figures are modeled after those in V. Petersen, "The Three Lunar Models of Ptolemy", Centaurus, 12 (1969) 142-171. This paper explains all the details of the full lunar model found in the Almagest.

${ }^{5}$ D. Pingree, ibid. (ref. 1-2), and references therein; B. L. van der Waerden, "The heliocentric system in Greek, Persian, and Indian astronomy", in From deferent to equant: a volume of studies in the history of science in the ancient and medieval near east in honor of E. S. Kennedy, Annals of the New York Academy of Sciences, 500 (1987), 525-546, and references therein.

${ }^{6}$ Such models with oscillating eccentricity permeate Indian texts, of course, and this is the first case I know of that we might finally understand the background of such things. ${ }^{7}$ S. B. Gaythorpe, "On Horrocks's Treatment of the Evection and the Equation of Center, with a Note on the Elliptic Hypotheses of Albert Curtz and its Correction by Boulliau and Newton", Monthly Notices of the Royal Astronomical Society, 85 (1925) 858-65.

${ }^{8}$ It might be that this model is foreshadowed in Brahmagupta's Brahmashputasiddhanta (ca. A.D. 628), which also has similar oscillations of the orbit elements for the Sun and Moon. See D. Pingree ibid. (ref. 1) p $569-570$, but either the text or the translation is too uncertain to be sure what is meant.

${ }^{9}$ V. Roberts, "The Solar and Lunar Theory of Ibn ash-Shatir", Isis, 48 (1957) 428-432.

${ }^{10}$ O. Neugebauer, "On the Planetary Theory of Copernicus", Vistas in Astronomy, 10 (1968) 89-103.

${ }^{11}$ E. S. Kennedy, "Late Medieval Planetary Theory”, Isis, 57 (1966) 365-378; G. Saliba, A History of Arabic Astronomy, (New York, 1994).

${ }^{12} \mathrm{~B}$. L. van der Waerden, “Ausgleichspunkt, 'methode der perser', und indische planetenrechnung", Archive for history of exact sciences, 1 (1961), 107-121; D. W. Duke, "The equant in India: the mathematical basis of Indian planetary models", Archive for History of Exact Sciences, 59 (2005) 563-576.

${ }^{13} \mathrm{~V}$. Petersen, ibid. (ref. 4) p 160.

${ }^{14} \mathrm{C}$. Wilson, "On the Origin of Horrocks's Lunar Theory", Journal for the history of astronomy, 18 (1987), 77-94; C. Wilson, "Predictive Astronomy in the century after Kepler", in Taton and Wilson, General History of Astronomy, 2A (1989) 161-206.

${ }^{15} \mathrm{C}$. Wilson, "Newton on the Moon's Variation and Apsidal Motion", in Buchwald and Cohen, Isaac Newton's Natural Philosophy, (Cambridge, 2001).

${ }^{16}$ D. W. Duke, "Computer Animations of Greek and Arabic Planetary Models", Journal for the History of Astronomy, 35, 225-228 (2004). 